\title{
Normal planar undulators doubling as transverse gradient undulators
}

\author{
Qika Jia* and Heting $\mathrm{Li}^{\dagger}$ \\ National Synchrotron Radiation Laboratory, University of Science and Technology of China, \\ Hefei 230029, Anhui, People's Republic of China
}

(Received 21 December 2016; published 21 February 2017)

\begin{abstract}
The transverse gradient undulator (TGU) has important application in the short-wavelength high-gain free electron lasers (FELs) driven by laser-plasma accelerators. However, the usual transversely tapered TGUs need special design and manufacture, and the transverse gradient cannot be tuned arbitrarily. In this paper we explore a new and simple method of using the natural transverse gradient of a normal planar undulator to compensate the beam energy spread effect. In this method, a vertical dispersion on the electron beam is introduced, then the dispersed beam passes through a normal undulator with a vertical off-axis orbit where the vertical field gradient is selected properly related to the dispersion strength and the beam energy spread. Theoretical analysis and numerical simulations for self-amplified spontaneous emission FELs based on laser plasma accelerators are presented, and indicate that this method can greatly reduce the effect of the beam energy spread, leading to a similar enhancement on FEL performance as the usual transversely tapered TGU, but with the advantages of economy, tunable transverse gradient and no demand of extra field for correcting the orbit deflection induced by the field gradient.
\end{abstract}

DOI: 10.1103/PhysRevAccelBeams.20.020707

\section{INTRODUCTION}

In recent years, laser plasma accelerators (LPAs) have made dramatic progress in producing high-energy and high-brightness electron beams, and have been pursued to drive compact $\mathrm{x}$-ray free electron lasers (FELs) [1-4]. However, currently the electron beam from LPA has large energy spread, typically on a few percent level. Such energy spread degrades the FEL gain and hinders the applications of LPAs in the short-wavelength FELs.

To understand the effect of the energy spread on FELs, we write the well-known resonant condition,

$$
\lambda_{s}=\frac{\lambda_{u}}{2 \gamma^{2}}\left(1+\frac{K^{2}}{2}\right)
$$

where $\gamma$ is the Lorentz factor of electron, and $\lambda_{u}, K$ are the period and the deflection parameter of the undulator, respectively. Obviously the energy spread will lead to a spread of the resonant wavelength and degrade the FEL gain. It is generally required that the FEL bandwidth broadening due to the energy spread should be smaller than the intrinsic bandwidth, which is proportional to the FEL parameter $\rho$ for high gain case and to the reciprocal of undulator period number for low gain case.

\footnotetext{
*Jiaqk@ustc.edu.cn

'liheting@ustc.edu.cn
}

Published by the American Physical Society under the terms of the Creative Commons Attribution 4.0 International license. Further distribution of this work must maintain attribution to the author(s) and the published article's title, journal citation, and DOI.
Therefore, the concept of using a "transverse gradient wiggler (undulator)", which was initially proposed to reduce the effects of large beam energy spread in low gain FELs [5,6], has been reconsidered to improve the performance of short-wavelength FELs driven by LPAs [3,7]. Meanwhile, it is also proposed using transverse gradient undulators (TGUs) to enhance the harmonic bunching in the seeded FEL regime [8], to suppress the microbunching instability [9], to improve the performance of ultimate storage rings based FEL [10], and to generate largebandwidth X-ray FEL $[11,12]$.

The transverse gradient undulator has a field strength that varies with transverse position, by properly correlating the energy with transverse position of the electrons, such that those with higher energy see a stronger field than those with lower energy, then the resonance condition [Eq. (1)] can be satisfied for all electrons. The transverse gradient of a TGU is defined as

$$
\alpha=\frac{\Delta K / K_{0}}{\Delta x},
$$

where $K_{0}$ is the strength parameter at the position of the electrons with the central energy. To make the resonant condition invariant for the electron beam with an energy spread $\Delta \gamma / \gamma$, from Eq. (1), the magnetic field should vary as

$$
\frac{\Delta K}{K_{0}}=\left(1+\frac{2}{K_{0}^{2}}\right) \frac{\Delta \gamma}{\gamma}
$$

Then the transverse dispersion strength should satisfy

$$
D=\frac{\Delta x}{\Delta \gamma / \gamma}=\left(1+\frac{2}{K_{0}^{2}}\right) \frac{1}{\alpha} .
$$




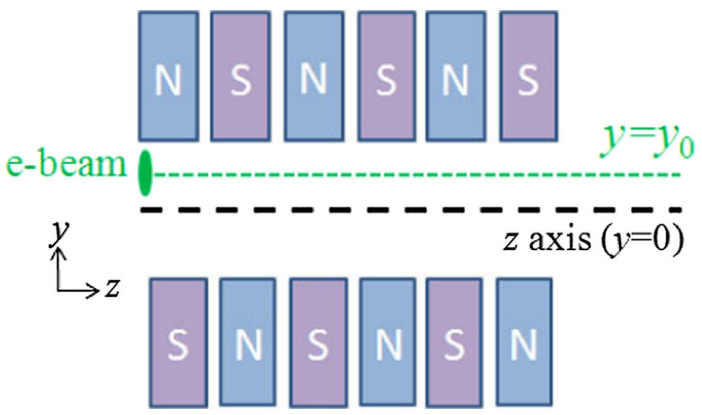

FIG. 1. Illustration for using the natural transverse gradient of a normal undulator to overcoming the effect of large energy spread by deviating the electron beam orbit of $y_{0}$. The electron beam is previously dispersed in the vertical direction.

The expansion of transverse beam size after dispersion should be moderate. It cannot be too large, otherwise the optical field gain will be depressed and multiple transverse modes will be developed [13]. On the other hand it must be sufficiently larger than the original size to generate linear dependence of electron energy on the transverse position.

Usually the transverse gradient of undulator field is technically realized by canting the magnetic poles, namely varying the undulator gap transversely, which results in an approximately linear horizontal dependence of the undulator field [13]. Such TGUs are the transversely tapered undulators. Other possible TGU geometries also have been discussed in Refs. [14,15] particularly for superconducting TGUs. Since the canting angle of a transversely tapered TGU is fixed, for a given resonant wavelength, the transverse field gradient is also fixed, and so is the dispersion strength from Eq. (4).

In this paper, we explore a new and simple method that a normal undulator doubles as a TGU for compensating the beam energy spread effect by using the natural transverse field gradient. For a planar undulator, as illustrated in Fig. 1, when a vertically dispersed electron beam passes through the undulator at an off-axis orbit, it will see a magnetic field with the natural vertical gradient. If Eq. (4) is satisfied in the vertical direction, the beam energy spread effect can be reduced and the FEL performance can be greatly improved. Theoretical analysis of this method is described in Sec. II. Then, simulation results for self-amplified spontaneous emission (SASE) FELs based on typical LPA beam are presented in Sec. III. Finally, we summarize in Sec. IV.

\section{METHODS}

A normal undulator usually has a vertical magnetic field. In the vertical direction the peak field $B_{y}$ varies as [16]

$$
B_{y}=B_{m} \cosh \left(k_{u} y\right)
$$

where $B_{m}$ is the peak field on the $z$ axis and $k_{u}$ is the wave number of the undulator. The variation of the undulator

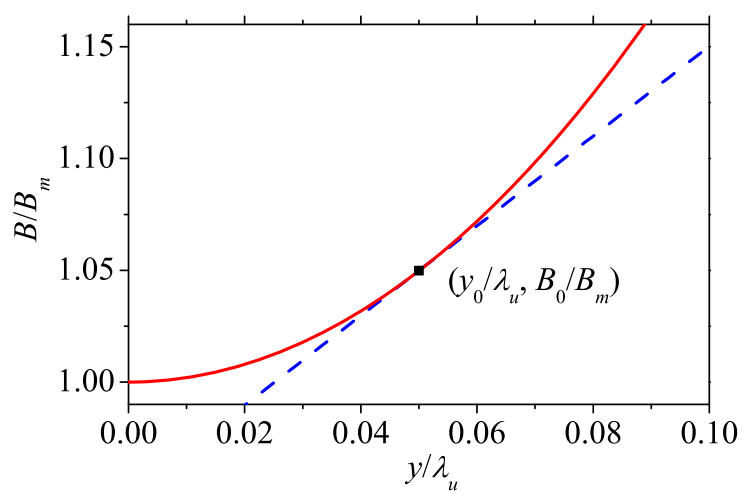

FIG. 2. The variation of the undulator peak field in vertical direction (solid red) and an example of linearly differential approximation (dashed blue).

field with vertical coordinate can be seen in Fig. 2. With the relation of $K=93.4 B_{y} \lambda_{u}$, the variation of $K$ parameter along the vertical coordinate is

$$
K(y)=K_{m} \cosh \left(k_{u} y\right) .
$$

Here, $K_{m}$ means the deflection strength on the $z$ axis.

For the off-axis orbit of $y=y_{0}$ (see Fig. 1), the natural vertical gradient $\alpha_{y}$ can be deduced as

$$
\alpha_{y}=\frac{\Delta K / K_{0}}{\Delta y}=k_{u} \tanh \left(k_{u} y_{0}\right) \approx k_{u}^{2} y_{0} .
$$

The approximation of the last step in the above equation is due to $k_{u} y_{0}<1$. From Eq. (7) we can see that, for a normal undulator, the natural transverse gradient is dependent on the vertical position of the electron beam center, but independent of the magnetic pole gap. Therefore by adjusting the injection position of the electron beam the natural vertical gradient can be adjusted. This is a distinct feature comparing with a usual transversely tapered TGU.

Another advantage of a normal undulator doubling as a TGU is that it does not need an extra correcting field. For a usual transversely tapered TGU, the electrons' wiggling and the magnetic field gradient are in the same plane, so that the electrons will see the field gradient when they oscillate in the undulator, hence the electrons will have a net deflection and an additional correcting field is required to correct the deflection [9]. However, for using the natural gradient, due to that the two directions are perpendicular to each other, the electrons will not see the field gradient. Therefore, the natural gradient induces no deflection of the electron beam.

While in the vertical plane the longitudinal field $\left(B_{z}\right)$ of the undulator focuses the off-axis electrons and makes them experience a betatron oscillation. Such effect is the socalled natural focusing, which may have an impact on the matching of the electron energy with the magnetic field and 
the gradient. If the vertical betatron wavelength is much longer than the undulator length $L_{u}$,

$$
\lambda_{\beta y}=\sqrt{2} \lambda_{u} \gamma / K \gg L_{u},
$$

then the natural focusing effect can be neglected. Otherwise, the betatron oscillation will gradually destroy the linear vertical energy chirp of the electron beam and degrade the final FEL performance. For this case, one should consider to add external focusing to counteract the natural focusing.

Note that the linear approximation of the field variation is available only for a small range of $\Delta y$ around $y_{0}$ (see Fig. 2). If $\Delta y$ is too large, the contribution of the nonlinear term to the field variation cannot be neglected. From the second differential term of the Taylor's expansion, it is

$$
\frac{\delta B}{B}=\frac{1}{2} k_{u}^{2} \Delta y^{2}=2 \pi^{2}\left(\frac{\Delta y}{\lambda_{u}}\right)^{2} .
$$

One can see that it is independent of the vertical position. For compensating the energy spread effect, we require that the bandwidth due to the field error should be smaller than the intrinsic bandwidth $(\Delta \lambda / \lambda)_{0}$, namely,

$$
\frac{\Delta \lambda}{\lambda}=\frac{K_{0}^{2}}{2+K_{0}^{2}} \frac{\delta B}{B}<\left(\frac{\Delta \lambda}{\lambda}\right)_{0} .
$$

For a high gain FEL, the intrinsic bandwidth scales to the pierce parameter $\rho$ [17]. Therefore combining Eq. (9) and Eq. (10), we give the applicable range of the linear approximation of the field gradient

$$
|\Delta y|<\frac{1}{k_{u}} \sqrt{2 \rho\left(1+\frac{2}{K_{0}^{2}}\right)} .
$$

This gives the upper limit of the vertical beam size after dispersion.

Typically the pierce parameter $\rho \sim 10^{-3}$ for soft X-ray FEL, $K=1-2$, and $\lambda_{u} \sim$ a few centimeters, thus for an offset beam orbit of a few millimeters, the vertical gradient can reach several hundreds per meter. And from Eq. (11) the applicable range of the linear approximation of the vertical gradient is $|\Delta y| \sim 10^{-2} \lambda_{u}$, corresponding to about several hundred microns. Comparing with the transversely tapered TGUs, whose field is very close to linearity though not strictly linear along the transverse position [18], the applicable range of linear approximation of the natural gradient is not so wide, but is sufficiently large for LPA beam due to its small transverse beam size. The situation will be better for an undulator with a long period.

\section{SIMULATION}

To validate our analysis above, we simulated a soft $\mathrm{X}$ ray SASE FEL based on typical LPA electron beam by using natural transverse gradient of a normal undulator as a TGU. In the simulation, the electron beam and undulator parameters considered in Ref. [3] are utilized as a representative example. They are as follows: a flattop electron beam with beam energy of $1 \mathrm{GeV}$, peak current of $10 \mathrm{kA}$, full width of $5 \mathrm{fs}$, relative energy spread of $1 \%$ and transverse emittance of $0.1 \mu \mathrm{m}$, and an undulator with period of $10 \mathrm{~mm}$ and total length of $5 \mathrm{~m}$.

The same as Ref. [3], we consider to generate $3.9 \mathrm{~nm}$ soft $\mathrm{x}$-ray radiation. The undulator strength for the center beam energy is $K_{0}=2$ and the vertical orbit offset is selected to be $y_{0}=0.5 \mathrm{~mm}$. According to the Eq. (7) and Eq. (4), the vertical field gradient and the dispersion strength are $\alpha_{y}=197.4 \mathrm{~m}^{-1}$ and $D=7.6 \mathrm{~mm}$, respectively. And from Eq. (11), the vertical beam size after dispersion should be smaller than $210 \mu \mathrm{m}$. Reasonably assuming the beta-function of $\beta=L_{u} / 2=2.5 \mathrm{~m}$, we get the initial transverse beam size of $\sigma_{x}=\sigma_{y}=11.3 \mu \mathrm{m}$. After vertical dispersion, the vertical beam size grows to $\sigma_{y}=76 \mu \mathrm{m}$. Such vertical size satisfies the requirement described in Eq. (11).

With the three-dimension code GENESIS [19], which already includes the vertical field gradient as well as the diffraction and transverse modes effects, we simulated the FEL process based on above parameters. Figure 3 shows the FEL power around $3.9 \mathrm{~nm}$ for using natural transverse gradient (NTG) with and without external focusing. The power of normal SASE is also given for comparison. One can find that, by using the natural gradient without any external focusing, the SASE power is enhanced by more than one order of magnitude and saturates within 5-m undulator. Such enhancement is a little lower than that of using TGU as presented in Ref. [3], owing to the

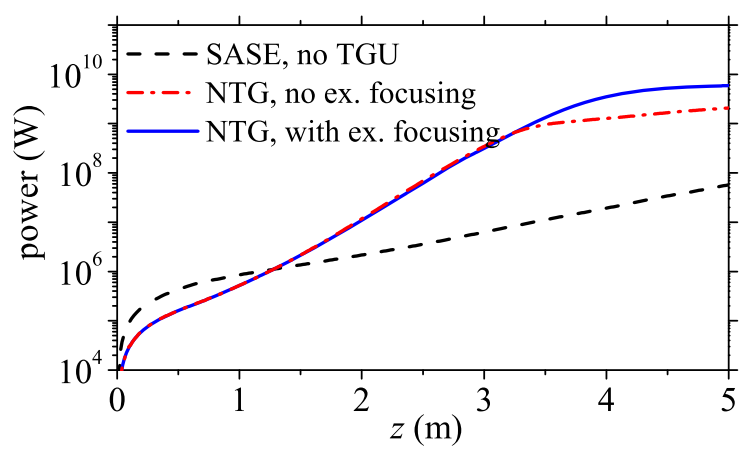

FIG. 3. FEL power around $3.9 \mathrm{~nm}$ for using natural transverse gradient (NTG, $y_{0}=0.5 \mathrm{~mm}$ ) with (solid blue) and without (dashed dotted red) external focusing, comparing with normal SASE (dashed black). The external focusing is realized by adding two quadrupoles focusing in $\mathrm{x}$ plane ahead each of two $2.5-\mathrm{m}$ undulator sections. 


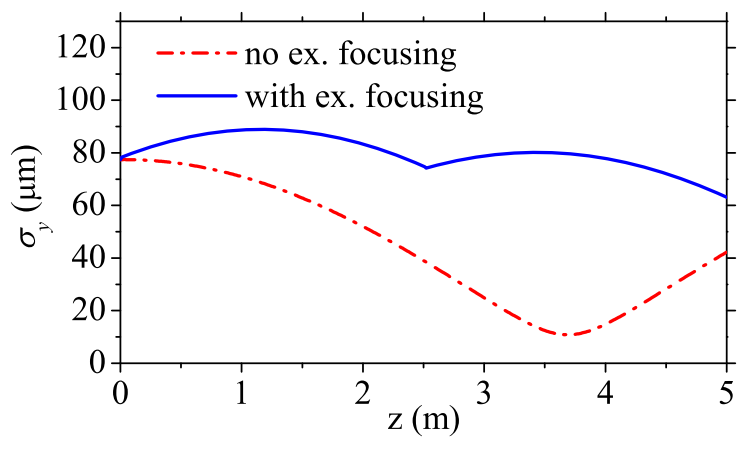

FIG. 4. The evolution of vertical beam size along the 5-m undulator for using natural transverse gradient with (solid blue) and without (dashed dotted red) external focusing.

vertical natural focusing of the undulator field. As shown in Fig. 4, the vertical beam size oscillates with a period of about $7 \mathrm{~m}$ and a peak-to-peak amplitude of about $70 \mu \mathrm{m}$. This oscillation will lead to a large variation of the energy chirp rate in vertical direction, namely the initial vertical energy dependence $\Delta \gamma / \Delta y=\gamma_{0} / D$ gradually changes along the longitudinal coordinate, which makes Eq. (4) not satisfied in the latter half of the $5-\mathrm{m}$ undulator. Therefore, the power growth rate tends to gradually decrease resulting in the earlier saturation at a relatively lower level.

To overcome the effect of the natural focusing, we added two quadrupoles ahead each of two 2.5-m undulator sections, which focused in the $x$ plane with focusing gradients of about $4 \mathrm{~T} / \mathrm{m}$ and $8 \mathrm{~T} / \mathrm{m}$, respectively, and an equal length of $20 \mathrm{~cm}$. For this case, the curves of the power and the vertical beam size are shown in Fig. 3 and Fig. 4, respectively. Note that, for more direct comparison, the evolutions of power and vertical beam size in the quadrupoles were omitted in these two figures. We can find that the external focusing makes the vertical oscillation

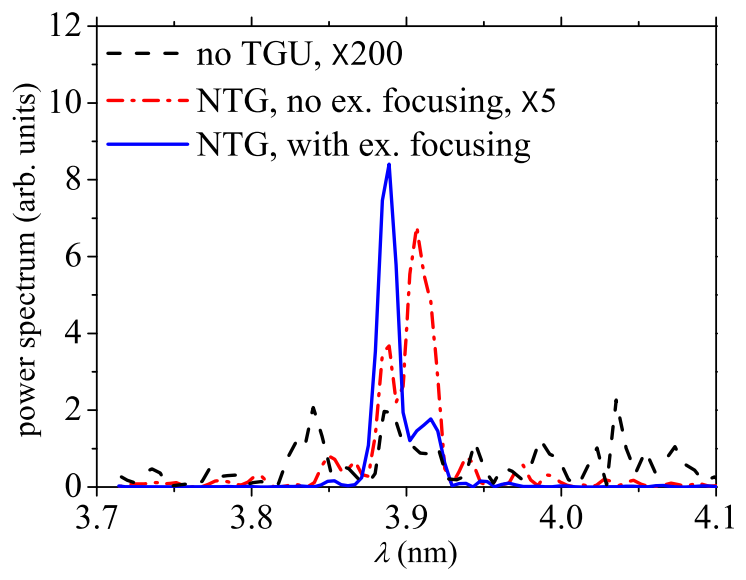

FIG. 5. Typical single-shot spectra around $3.9 \mathrm{~nm}$ of normal SASE FELs (dashed black), for using natural transverse gradient with (solid blue) and without (dashed dotted red) external focusing.
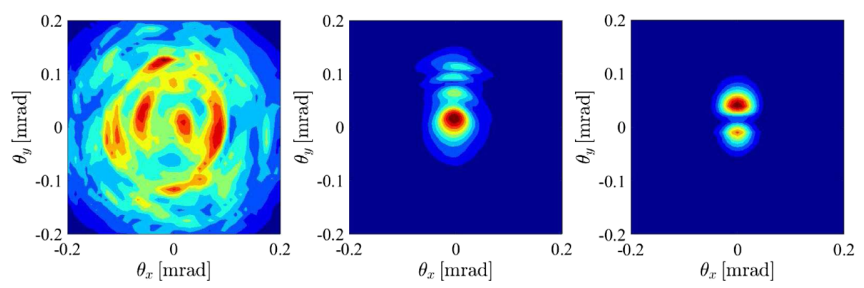

FIG. 6. Transverse mode pattern around $3.9 \mathrm{~nm}$ of normal SASE FELs (left), for using natural transverse gradient without external focusing (middle), and for NTG with external focusing (right).

amplitude of the beam size smaller. The FEL power grows continuously within the full length of the 5-m undulator and is enhanced by about two orders of magnitude.

Figure 5 shows the typical single-shot spectra of the three cases. One can see that the longitudinal coherence of the normal SASE is very poor with a rms bandwidth of about $2 \%$ while a single coherent spike is generated by using natural transverse gradient. The transverse mode pattern for the three cases are also given in Fig. 6. The transverse coherence also can be greatly improved by using natural transverse gradient. These simulation results are almost the same as those of using usual transversely tapered TGUs presented in Ref. [3].

The most different point of using natural gradient comparing with usual TGUs is that we should accurately control the beam orbit offset. Tolerance of the FEL power on the beam orbit offset for the example above has been studied and given in Fig. 7. The result shows that, for using natural transverse gradient with external focusing, if requiring $80 \%$ of the power for the case of $y_{0}=0.5 \mathrm{~mm}$ remains, the accuracy of the orbit should be better than $30 \mu \mathrm{m}$, which can be easily satisfied by modern technology of beam measurement and control.

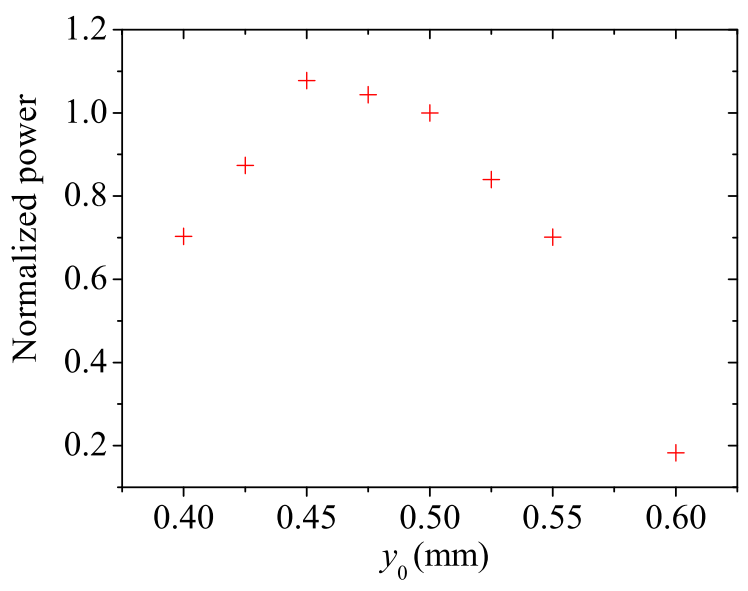

FIG. 7. The variation of the FEL power with the offset of the vertical beam orbit for using natural transverse gradient with external focusing. 


\section{SUMMARY}

In summary, we proposed a new and simple method of using the natural transverse gradient of a normal planar undulator to reduce the effect of the large beam energy spread. The significant enhancement has been demonstrated with theoretical analysis and 3D simulations. In general, with this method one can achieve the similar results as using a usual transversely tapered TGU. Furthermore, a normal planar undulator doubling as a TGU has several distinct advantages. The main advantage first is very economical and convenient since the existing undulators can be directly used as TGUs. Second, the natural transverse gradient can be adjusted conveniently in a quite large range by changing the beam orbit offset or controlling the top and bottom magnetic poles moving independently, while the usual transversely tapered TGU is used for special parameters sets, and there may be some problem when it is used as a normal undulator. Because when the beam energy spread is small, the transverse gradient may lead to bad performance, such as gain degradation, large bandwidth, and so on. Lastly, using the natural transverse gradient does not need an extra correcting magnetic field because the natural gradient induces no deflection of the electron beam.

However, using the natural gradient also has disadvantages, for instance, the linear range of the natural gradient field is rather small so that a smaller beam size and a larger undulator period are preferred. In addition, since the natural gradient and the natural focusing are in the same direction, external focusing is required to counteract the effects of the natural focusing. when the undulator length is close to or longer than the betatron wavelength of the natural focusing.

Though in this paper, we just took LPA based FEL as an example of the application of using the natural transverse gradient, we think that this concept may also be used for other purposes of TGUs, such as ultimate storage ring based FEL, phase-merging harmonic generation (PEHG) FEL, and so on. And we expect the study presented here will promote the application of using the natural transverse gradient in experiments.

\section{ACKNOWLEDGMENTS}

This work was supported by the National Natural Science Foundation of China under Grants No. 11375 199, No. 11205156, No. 11675178, and National Key Research and Development Program of China (No. 2016YFA0401901).

[1] E. Esarey, C. Schroeder, and W. Leemans, Physics of laserdriven plasma-based electron accelerators, Rev. Mod. Phys. 81, 1229 (2009).

[2] M.P. Anania et al., An ultrashort pulse ultra-violet radiation undulator source driven by a laser plasma wakefield accelerator, Appl. Phys. Lett. 104, 264102 (2014).
[3] Z. Huang, Y. Ding, and C. B. Schroeder, Compact X-ray Free-Electron Laser from a Laser-Plasma Accelerator Using a Transverse-Gradient Undulator, Phys. Rev. Lett. 109, 204801 (2012).

[4] I. V. Pogorelsky, M. N. Polyanskiy, and W. D. Kimura, Mid-infrared lasers for energy frontier plasma accelerators, Phys. Rev. Accel. Beams 19, 091001 (2016).

[5] T. I. Smith, L. R. Elias, J. M. J. Madey, and D. A. G. Deacon, Reducing the sensitivity of a free-electron laser to electron energy, J. Appl. Phys. 50, 4580 (1979).

[6] N. Kroll, P. Morton, M. Rosenbluth, J. Eckstein, and J. Madey, Theory of the transverse gradient wiggler, IEEE J. Quantum Electron. 17, 1496 (1981).

[7] M E Couprie et al., An application of laser-plasma acceleration: towards a free-electron laser amplification, Plasma Phys. Controlled Fusion 58, 034020 (2016).

[8] Haixiao Deng and Chao Feng, Using Off-Resonance Laser Modulation for Beam-Energy-Spread Cooling in Generation of Short-Wavelength Radiation, Phys. Rev. Lett. 111, 084801 (2013).

[9] Chao Feng, Haixiao Deng, Dong Wang, and Zhentang Zhao, Phase-merging enhanced harmonic generation freeelectron laser, New J. Phys. 16, 043021 (2014).

[10] Y. Ding, P. Baxevanis, Y. Cai, Z. Huang, and R. Ruth, Highgain $\mathrm{X}$-ray FELs using a transverse gradient undulator in a diffraction-limited storage ring, in Proceedings of the 4th International Particle Accelerator Conference, IPAC-2013, Shanghai, China, 2013 (JACoW, Shanghai, China, 2013), p. 2286.

[11] A. Saa Hernandez, E. Prat, S. Bettoni, B. Beutner, and S. Reiche, Generation of large-bandwidth x-ray free-electronlaser pulses, Phys. Rev. Accel. Beams 19, 090702 (2016).

[12] Eduard Prat, Marco Calvi, and Sven Reiche, Generation of ultra-large-bandwidth X-ray freeelectron-laser pulses with a transverse-gradient undulator, J. Synchrotron Radiat. 23, 874 (2016).

[13] P. Baxevanis, Z. Huang, R. Ruth, and C. B. Schroeder, Eigenmode analysis of a high-gain free-electron laser based on a transverse gradient undulator, Phys. Rev. ST Accel. Beams 18, 010701 (2015).

[14] G. Fuchert et al., A novel undulator concept for electron beams with a large energy spread, Nucl. Instrum Methods Phys. Res., Sect. A 672, 33 (2012).

[15] V. Afonso Rodriguez et al., Design optimization for a nonplanar undulator for the JETI-Laser wakefield accelerator in JENA, in Proceedings of the 2nd International Particle Accelerator Conference, San Sebastiáán, Spain (EPS-AG, Spain, 2011), p. 1452.

[16] K. Halbach, Design of permanent multipole magnets with oriented rare earth cobalt material, Nucl. Instrum. Methods Phys. Res. Sect. A 169, 1 (1980).

[17] R. Bonifacio, C. Pellegrini, and L. M. Narducci, Collective instabilities and high-gain regime in a free electron laser, Opt. Commun. 50, 373 (1984).

[18] Jiayu Li, Heting Li, Qika Jia, and Baiting Du, Calculation and analysis of the magnetic field of a transverse gradient undulator, in Proceedings of the 7th International Particle Accelerator Conference, Busan, Korea, 2016 (JACoW, Busan, Korea, 2016), p. 1130.

[19] S. Reiche, GENESIS 1.3: a fully 3D time-dependent FEL simulation code, Nucl. Instrum. Methods Phys. Res., Sect. A 429, 243 (1999). 